\title{
Subamostragem de pólen apícola para análise melissopalinológica
}

\author{
Anna Frida Hatsue Modro ${ }^{1,6}$, Emanuel Maia ${ }^{2}$, Izabel Christina da Silva ${ }^{3}$, Cynthia Fernandes Pinto da Luz ${ }^{4}$ \\ e Dejair Message ${ }^{5}$
}

Recebido: 06.10.2008; aceito: 19.11.2009

ABSTRACT - (Sub-sampling of bee pollen loads for melissopalynological analysis). The aim of this study was to evaluate various criteria of sub-sampling used in melissopalynological analysis of pollen loads collected by the africanized Apis mellifera (L.) honeybees. The experiment included four different sub-sample weights $(0,5 ; 1,0 ; 2,0 ; 5,0 \mathrm{~g})$, taken from 60 $\mathrm{g}, 100 \mathrm{~g}$ and $200 \mathrm{~g}$ samples of heterofloral pollen loads. The richness of pollen types was higher in the heavier sub-samples $(\mathrm{p}=0,006)$, however, the additional pollen types occurred with low representativity. The weight of the sub-sample did not influence the change of frequency class in the predominant pollen types $(p=0,263)$. There was a bigger shift of classification positions of the predominant pollen types with a reduction of the size of the pollen grain $(p=0,001)$. The results pointed to the use of sub-sample of $5 \mathrm{~g}$ for evaluation of the richness of the pollen types in heterofloral samples, however, sub-sample of $0,5 \mathrm{~g}$ did not limited the evaluation of the predominant pollen types.

Key words: Apis mellifera, bee pollen loads, sub-sampling criteria, botanical source

RESUMO - (Subamostragem de pólen apícola para análise melissopalinológica). Este trabalho teve por objetivo avaliar os vários critérios de subamostragens empregados na análise melissopalinológica de cargas de pólen coletadas por abelhas Apis mellifera (L.) africanizadas. O experimento considerou quatro diferentes pesos de subamostras $(0,5 ; 1,0 ; 2,0 \mathrm{e} 5,0 \mathrm{~g})$ retiradas de lotes de cargas de pólen heteroflorais de $60 \mathrm{~g}, 100 \mathrm{~g}$ e $200 \mathrm{~g}$. A riqueza de tipos polínicos foi maior em subamostras de maior peso $(\mathrm{p}=0,006)$, no entanto, tipos polínicos adicionais ocorreram com baixa representatividade. $O$ peso da subamostra não influenciou na mudança da classe de frequência dos principais tipos polínicos $(p=0,263)$. Houve um maior número de trocas de posições na classificação dos principais tipos polínicos com a redução do tamanho do grão de pólen ( $\mathrm{p}=0,001)$. Os resultados indicaram o uso de subamostragens de $5 \mathrm{~g}$ para estudos de avaliação da riqueza de tipos polínicos em amostras heteroflorais, entretanto, subamostragem de $0,5 \mathrm{~g}$ não limitou a avaliação dos principais tipos polínicos.

Palavras-chave: Apis mellifera, cargas de pólen, critérios de subamostragem, origem botânica

\section{Introdução}

O grão de pólen apresenta parede externa quimicamente estável e morfologicamente variada, o que permite através da análise melissopalinológica das cargas de pólen coletadas pelas abelhas, a identificação dos diversos tipos polínicos constituintes, a verificação dos períodos de produção e a determinação da origem botânica e geográfica das amostras (Salgado-Labouriau 1973, Barth 2004). Na análise polínica, os caracteres morfológicos dos grãos de pólen são apresentados por meio de descrições, empregando terminologia própria e ao mesmo tempo acompanhados por representações gráficas através de desenhos e fotomicrografias, identificando-se os tipos polínicos (Barth 1989). O termo "tipo polínico" é designado pelo nome de um dos gêneros ou espécie que nele se inclui, mas não está relacionado ao Código Internacional de Nomenclatura Botânica, e sim estabelece uma proximidade do material analisado a um determinado grupo taxonômico (SalgadoLabouriau 1973).

Dois métodos podem ser utilizados na análise polínica com vista à identificação dos tipos polínicos e sem o comprometimento do resultado final da análise, o método com acetólise (Erdtman 1960) e o método

1. Universidade de São Paulo, Escola Superior de Agricultura 'Luiz de Queiroz', Departamento de Entomologia, Fitopatologia e Zoologia Agrícola, Caixa Postal 9, 13418-900 Piracicaba, SP, Brasil

2. Universidade Federal de Rondônia, Departamento de Agronomia, Av. Norte Sul 7300, 76940-000 Rolim de Moura, RO, Brasil

3. Universidade de São Paulo, Faculdade de Filosofia Ciências e Letras de Ribeirão Preto, Departamento de Entomologia, Av. Bandeirantes 3900, 14040-901 Ribeirão Preto, SP, Brasil

4. Instituto de Botânica, Centro de Pesquisa em Plantas Vasculares, Av. Miguel Estéfano 3687, 04301-902 São Paulo, SP, Brasil

5. Universidade Federal de Viçosa, Departamento de Biologia Animal, 36570-000 Viçosa, MG, Brasil

6. Autor para correspondência: fridamodro@gmail.com 
direto ou sem acetólise (Maurizio \& Louveaux 1965). No entanto, para análise melissopalinológica dos produtos apícolas o método direto permite caracterizar melhor as amostras, pois não remove óleos e trifina existentes nos grãos de pólen (muito comuns nos tipos entomófilos), não muda a cor da parede externa, não destrói o citoplasma e nem os elementos figurados como bactérias e leveduras, características essas que auxiliam na identificação dos tipos polínicos e na certificação da qualidade desse produto vendido no comércio (Barth 1989, Luz et al. 2007a).

As amostragens adotadas para bolotas ou cargas de pólen apícola em amostras heteroflorais da região neotropical não seguem uma padronização e, geralmente, consistem na retirada dos lotes originais de subamostras com peso úmido variando entre $2 \mathrm{e}$ $5 \mathrm{~g}$, sem considerar o peso total dos grãos de pólen (Almeida-Muradian et al. 2005, Luz et al. 2007b, Modro et al. 2007).

Considerando, a importância da composição das cargas de pólen de abelhas Apis mellifera (L.) tanto para consumo humano como para fins de pesquisa científica, o objetivo deste trabalho foi avaliar diferentes pesos de subamostras heteroflorais usualmente empregados na análise melissopalinológica, quanto à representatividade e riqueza dos tipos polínicos.

\section{Material e métodos}

O pólen heterofloral utilizado foi obtido entre 12 de agosto e 13 de dezembro de 2005, em coletores de pólen instalados em cinco colméias de Apis mellifera localizadas na região de Viçosa, Minas Gerais, Brasil. O pólen coletado foi homogeneizado e dividido em três grupos, a saber, $60 \mathrm{~g}, 100 \mathrm{~g}$ e $200 \mathrm{~g}$. Foram retiradas, de forma aleatória destas amostras, subamostras de $5 \mathrm{~g}, 2 \mathrm{~g}, 1 \mathrm{~g}$ e $0,5 \mathrm{~g}$ de cada grupo, constituindo assim 12 tratamentos. Em cada tratamento foram realizadas quatro repetições, e cada unidade experimental foi constituída da média dos valores observados em cinco lâminas de microscopia. A preparação melissopalinológica das amostras foi realizada conforme o método padrão europeu de Maurizio \& Louveaux (1965), adaptado para cargas de pólen por Almeida-Muradian et al. (2005).

A identificação dos tipos polínicos foi baseada, principalmente, na coleção de referência do pólen das plantas floridas na região de estudo e em catálogos palinológicos (Salgado-Labouriau 1973,
Barth 1989, Roubik \& Moreno 1991, Melhem et al. 2003). Foram identificados e contados por varredura aproximadamente 300 grãos de pólen por lâmina, com um total de 1500 grãos por repetição.

As variáveis avaliadas foram: riqueza (total de tipos polínicos), média da freqüência relativa e da posição ocupada pelos principais tipos polínicos (frequência $>2 \%$ ). A importância dos tipos polínicos foi avaliada por um ordenamento, baseado na frequiência dos tipos polínicos com valor superior a $2 \%$. Posteriormente, para avaliar o número de vezes que houve troca de posição na classificação do tipo polínico, utilizou-se da estimativa do erro quadrático médio calculado entre a classificação média do tipo polínico e a sua classificação por peso de cada amostra. A relação entre a freqüência acumulada e a ordem dos tipos polínicos foi avaliada por regressão. As demais variáveis foram submetidas à análise de regressão e correlação linear. Todas as análises foram realizadas com o auxílio do software estatístico R (R Core Team 2006).

\section{Resultados e Discussão}

O número máximo de tipos polínicos encontrados em uma única lâmina foi 21 , sendo que o total de tipos polínicos encontrados em todas as lâminas foi 38. O número médio de tipos polínicos encontrados em uma unidade experimental foi 18 , no entanto, tipos polínicos adicionais, ocorreram com baixa representatividade na amostragem (figura 1). A equação ajustada entre a frequiência acumulada e a ordem do tipo polínico corroborou o fato de que, tipos polínicos com frequiências menores que $2 \%$ nas cargas de pólen heteroflorais, são provenientes de espécies de plantas subrepresentadas nos espectros polínicos, pois oferecem pouco pólen para as abelhas e, são classificados como Pólen Isolado Ocasional (Barth 1989). Não foi observado efeito da amostra sobre a riqueza de tipos polínicos $(\mathrm{p}=0,460)$, garantindo, juntamente com a análise qualitativa dos tipos polínicos, as mesmas condições entre as diferentes subamostragens realizadas.

O peso da subamostra e a riqueza apresentaram o coeficiente angular da regressão positivo e significativo $(p=0,006)$. Ao estratificar as subamostras em relação ao peso da amostra, compondo um intervalo de subamostragem entre $0,25 \%$ e $8,33 \%$, os resultados confirmaram o efeito positivo do peso da amostra 


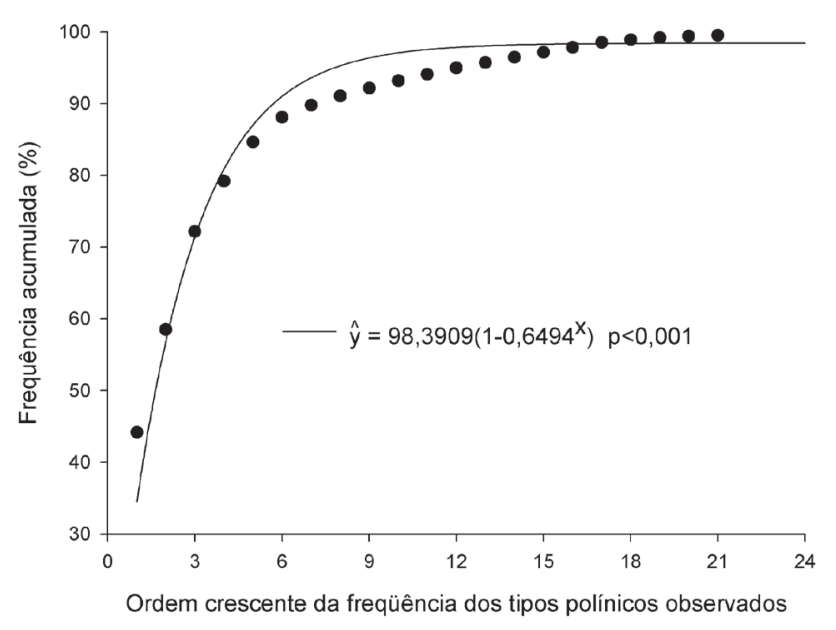

Figura 1. Dispersão entre a frequência acumulada e a ordem crescente da freqüência dos tipos polínicos encontrados em amostras de cargas de pólen heteroflorais.

Figure 1. Dispersion between the cumulative frequency and the increasing frequency order of pollen types in heterofloral pollen load samples.

em relação à riqueza dos tipos polínicos (figura 2). Desta maneira, recomenda-se o uso de amostras de $5 \mathrm{~g}$ para a determinação da riqueza de tipos polínicos em amostras de cargas de pólen heteroflorais, contudo, as subamostras de $2 \mathrm{~g}$ são as mais compatíveis com a preparação melissopalinológica, pois normalmente são utilizados tubos de centrífuga cônicos e de $15 \mathrm{~mL}$ de volume, uma vez que não acarretam em uma excessiva deposição do sedimento polínico no fundo dos mesmos.

Do total de tipos polínicos apenas seis apresentaram proporção maior que $2 \%$, representando juntos $88,12 \%$ do total da amostra, sendo em ordem descrescente de importância Baccharis, Myrcia, Vernonia, Scrophulariaceae, Cecropia e Gochnatia (tabela 1). As frequiências de Myrcia e Vernonia foram influenciadas pelo peso da amostra (tabela 2). Apesar do peso da amostra ter alterado significativamente apenas a frequiência desses dois tipos polínicos, observou-se que houve a troca de posições com maior intensidade entre os tipos polínicos menos freqüentes, com exceção de Cecropia (tabela 3). Todavia esta variação não apresentou correlação com a proporção subamostrada ( $\mathrm{Q}=-0,147 ; \mathrm{p}=0,263)$, deste modo a utilização de subamostras de $0,5 \mathrm{~g}$ pode ser adotada para determinar os principais tipos polínicos ocorrentes em uma amostra de cargas de pólen heteroflorais.

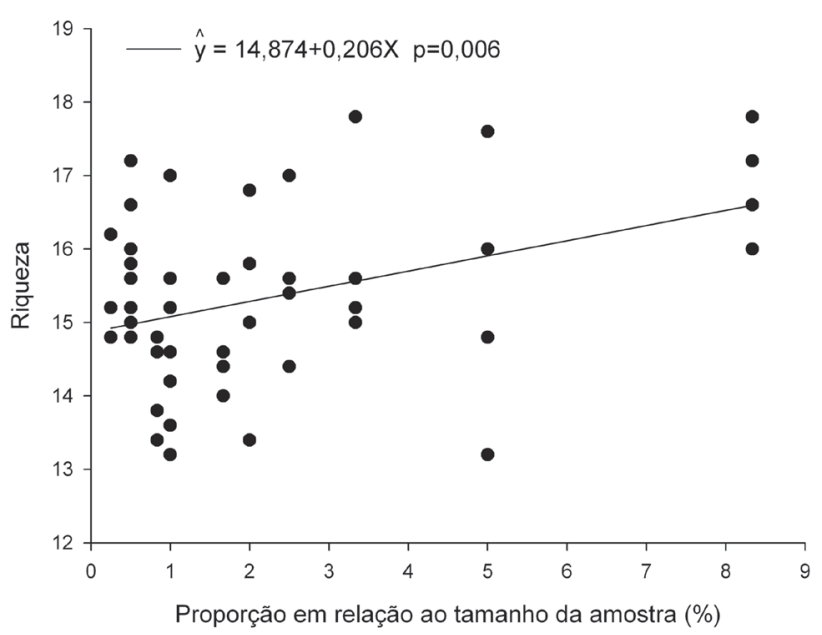

Figura 2. Dispersão entre a riqueza (total de tipos polínicos) e a proporção subamostrada encontrados em amostras de cargas de pólen heteroflorais.

Figure 2. Dispersion between the richness (total of pollen types) and the sub-sample ratio in heterofloral pollen load samples

Com o intuito de compreender se o tamanho do grão de pólen está relacionado com a variação relatada acima, procedeu-se um estudo correlacionando as medidas de diâmetro equatorial e polar do pólen com a estimativa do erro quadrático médio. Assim, com a redução do tamanho do diâmetro equatorial ( $\varrho=-0,392 ; p=0,001)$ ou polar $(\varrho=-0,471 ; p=0,001)$ verificou-se um aumento na troca de posições da classificação dos principais tipos polínicos. A influência do volume do pólen para estimar a sua importância relativa nas análises melissopalinológicas foi proposta por Tasei (1973) e posteriormente avaliada por Silveira (1991) e Biesmeijer et al. (1992), sendo ressaltada em Castagnino et al. (2004) e Modro et al. (2007) a necessidade dessa avaliação de volume para uma correta interpretação da amostra, principalmente para atender aos interesses comerciais da apicultura quanto à definição da origem botânica e das reais proporções desse recurso floral nos lotes de pólen apícola comercializados.

Conclui-se que com o aumento do peso da subamostragem de cargas de pólen heteroflorais, houve maior riqueza de tipos polínicos.

O peso da subamostra não influenciou no ordenamento dos principais tipos polínicos. 
Tabela 1. Valores percentuais médios dos tipos polínicos observados em uma amostra homogeneizada de pólen apícola heterofloral, coletado entre 12 de agosto e 13 de dezembro de 2005, em Viçosa, Minas Gerais, Brasil.

Table 1. Average percentage values of pollen types in one homogenized heterofloral pollen load sample, collected between August 12 and December 13 in 2005, Viçosa, Minas Gerais, Brazil.

\begin{tabular}{|c|c|c|c|c|c|c|c|c|c|c|c|c|}
\hline \multirow{3}{*}{ Tipos polínicos } & \multicolumn{12}{|c|}{ Tratamentos } \\
\hline & \multicolumn{4}{|c|}{$60 \mathrm{~g}$} & \multicolumn{4}{|c|}{$100 \mathrm{~g}$} & \multicolumn{4}{|c|}{$200 \mathrm{~g}$} \\
\hline & $0,5 \mathrm{~g}$ & $1 \mathrm{~g}$ & $2 \mathrm{~g}$ & $5 \mathrm{~g}$ & $0,5 \mathrm{~g}$ & $1 \mathrm{~g}$ & $2 \mathrm{~g}$ & $5 \mathrm{~g}$ & $0,5 \mathrm{~g}$ & $1 \mathrm{~g}$ & $2 \mathrm{~g}$ & $5 \mathrm{~g}$ \\
\hline Alchornea & 0,08 & - & - & 0,04 & 0,04 & - & 0,04 & - & 0,24 & 0,36 & 0,04 & 0,02 \\
\hline Amaranthaceae & - & - & - & - & 0,14 & - & - & 0,04 & - & - & 0,02 & 0,02 \\
\hline Anacardiaceae & 1,84 & 1,08 & 0,24 & 0,24 & - & 0,78 & 0,51 & - & 1,41 & 1,87 & 0,36 & 0,59 \\
\hline Araceae & 0,41 & 0,34 & 0,41 & 0,90 & 0,56 & 0,16 & 0,14 & 0,22 & 0,22 & 0,26 & 0,08 & 0,69 \\
\hline Arecaceae 1 & 2,10 & 0,95 & 1,16 & 0,86 & 0,68 & 0,78 & 0,57 & 0,48 & 0,83 & 0,52 & 0,53 & 0,67 \\
\hline Arecaceae 2 & 0,02 & - & 0,08 & 0,13 & 0,06 & 0,04 & 0,02 & 0,02 & 0,06 & - & 0,04 & 0,14 \\
\hline Arecaceae 3 & - & - & 0,16 & 0,13 & 0,02 & 0,02 & 0,21 & 0,38 & 0,20 & - & 0,20 & 0,04 \\
\hline Baccharis & 39,06 & 45,25 & 45,69 & 42,36 & 39,20 & 42,34 & 40,39 & 49,82 & 42,41 & 46,88 & 48,65 & 48,65 \\
\hline Caesalpinia & - & 0,02 & - & 0,13 & 0,06 & 0,02 & 0,04 & 0,04 & 0,02 & 0,04 & - & - \\
\hline Cecropia & 0,08 & 0,34 & 6,37 & 7,37 & 9,97 & 0,14 & 9,63 & 9,39 & 5,52 & 0,36 & 5,68 & 11,19 \\
\hline Celtis & 0,06 & 0,06 & 0,06 & 0,07 & - & - & 0,02 & - & 0,04 & 0,16 & 0,02 & 0,10 \\
\hline Cirsium & 0,16 & 0,44 & 0,39 & 1,01 & 1,67 & 2,27 & 1,09 & 0,40 & 0,42 & 0,18 & 0,65 & 0,20 \\
\hline Citrus & 0,29 & 0,38 & 0,39 & 0,33 & 0,12 & 0,20 & 0,29 & 0,32 & 0,12 & 0,04 & 0,26 & 0,49 \\
\hline Coffea & 1,48 & 0,82 & 0,53 & 0,86 & 0,80 & 0,84 & 0,51 & 0,38 & 1,13 & 0,58 & 0,34 & 0,16 \\
\hline Cuphea & 0,21 & 0,08 & 0,14 & 0,15 & 0,56 & 0,32 & 0,25 & 0,08 & 0,49 & 0,26 & 0,04 & 0,06 \\
\hline Datura & 0,02 & - & - & 0,04 & - & 0,02 & - & 0,02 & - & - & - & - \\
\hline Dombeya & 0,02 & - & - & - & 0,02 & - & 0,04 & 0,04 & - & 0,02 & - & 0,02 \\
\hline Elephantopus & 1,22 & 0,78 & 0,59 & 0,62 & 1,81 & 1,59 & 0,98 & 0,74 & 1,76 & 0,90 & 0,71 & 0,59 \\
\hline Eucalyptus & 1,26 & 2,42 & 1,73 & 1,54 & 0,92 & 1,00 & 1,91 & 1,10 & 1,62 & 2,45 & 1,42 & 2,18 \\
\hline Euphorbiaceae & 0,72 & 0,84 & 0,92 & 1,67 & 0,92 & 0,58 & 0,90 & 1,10 & 0,79 & 0,90 & 1,68 & 2,68 \\
\hline Fabaceae & 0,23 & 0,02 & 0,06 & 0,02 & - & - & - & - & 0,04 & - & - & - \\
\hline Ficus & - & - & - & - & 0,38 & - & - & 0,04 & - & 0,14 & - & 0,16 \\
\hline Gochnatia & 6,93 & 8,24 & 6,63 & 5,26 & 2,73 & 1,69 & 1,19 & 0,82 & 1,76 & 3,35 & 1,86 & 1,16 \\
\hline Guettarda & - & - & - & 0,02 & - & - & - & & & 0,02 & & 0,04 \\
\hline Myrcia & 16,62 & 17,08 & 8,27 & 7,92 & 15,83 & 24,00 & 18,07 & 10,01 & 18,21 & 16,56 & 13,03 & 6,37 \\
\hline Paullinia & - & 0,13 & - & - & - & - & - & - & - & - & - & - \\
\hline Poaceae & - & - & - & 0,04 & 0,02 & - & - & 0,04 & 0,06 & 0,06 & 0,10 & 0,04 \\
\hline Ricinus & 1,79 & 0,19 & 0,35 & 0,79 & 1,15 & 0,92 & 0,57 & 0,32 & 1,33 & 0,24 & 0,16 & 0,22 \\
\hline Scrophulariaceae & 4,68 & 1,98 & 9,59 & 3,74 & 8,08 & 7,65 & 10,29 & 6,32 & 5,33 & 12,39 & 10,05 & 4,98 \\
\hline Senecio & 2,99 & 0,97 & 0,86 & 0,66 & - & 0,52 & 0,29 & - & 0,85 & 0,82 & 0,16 & 0,41 \\
\hline Tabebuia & 0,06 & 0,65 & 0,43 & 0,59 & 3,21 & 1,32 & 0,96 & 1,04 & 0,26 & 0,54 & 1,50 & 0,14 \\
\hline Trema & 0,47 & 1,71 & 2,73 & 1,25 & 1,47 & 0,92 & 1,11 & 1,44 & 1,01 & 1,12 & 0,85 & 1,34 \\
\hline Vernonia & 17,18 & 15,12 & 12,18 & 21,07 & 9,48 & 11,62 & 9,96 & 15,24 & 13,80 & 8,81 & 11,55 & 16,53 \\
\hline Não identificado 1 & - & - & - & 0,02 & - & 0,06 & - & 0,04 & - & - & - & 0,02 \\
\hline Não identificado 2 & - & - & 0,02 & - & - & 0,08 & - & 0,04 & 0,06 & - & - & - \\
\hline Não identificado 3 & - & - & - & 0,09 & 0,12 & 0,10 & - & 0,06 & - & 0,06 & - & 0,08 \\
\hline Não identificado 4 & - & - & - & - & - & - & - & - & 0,02 & 0,08 & - & - \\
\hline Não identificado 5 & - & 0,11 & - & 0,07 & - & - & 0,02 & 0,04 & - & - & - & - \\
\hline Total (\%) & 100 & 100 & 100 & 100 & 100 & 100 & 100 & 100 & 100 & 100 & 100 & 100 \\
\hline
\end{tabular}


Tabela 2. Tipos polínicos mais freqüentes em amostras de cargas de pólen heteroflorais, coeficiente angular e valores de probabilidade associados ao teste $\mathrm{F}(\mathrm{P}>\mathrm{F})$ para regressão linear simples entre a frequiência do tipo polínico e o peso da amostra.

Table 2. The most frequent pollen types in heterofloral pollen load samples, the angular coefficient and values of probability associated to $\mathrm{F}(\mathrm{P}>\mathrm{F})$ test for simple linear regression between the frequency of the pollen type and the weight of the sample.

\begin{tabular}{lcc}
\hline Tipo polínico & Coeficiente angular & $\mathbf{P}>\mathbf{F}$ \\
\hline Baccharis & 0,3283 & 0,496 \\
Cecropia & 0,7111 & 0,153 \\
Gochnatia & 0,1377 & 0,452 \\
Myrcia & $-1,5127$ & 0,001 \\
Scrophulariaceae & $-0,4063$ & 0,232 \\
Vernonia & 1,0353 & $<0,001$ \\
\hline
\end{tabular}

Houve um maior número de trocas de posição da classificação entre os principais tipos polínicos com a redução do tamanho dos diâmetros equatorial e polar dos grãos de pólen.

Recomenda-se o uso de subamostras de $5 \mathrm{~g}$ para uma avaliação mais abrangente da riqueza dos tipos polínicos.

O uso de subamostras de $0,5 \mathrm{~g}$ é suficiente para a determinação dos principais tipos polínicos ocorrentes numa amostra de cargas de pólen heterofloral.

\section{Agradecimentos}

À Ângela Maria da Silva Corrêa do Instituto de Botânica (SP), pelo auxílio nas identificações polínicas e fotomicrografias. Ao funcionário do Apiário Central

Tabela 3. Erro quadrático médio para os principais tipos polínicos presentes em amostras de cargas de pólen heteroflorais, considerando as diferentes proporções de subamostragem em relação ao peso total da amostra.

Table 3. Average quadratic error for the main pollen types in heterofloral pollen load samples, considering the different ratios of subsampling in relation to the total weight of the sample.

\begin{tabular}{|c|c|c|c|c|c|c|c|c|c|c|}
\hline \multirow{2}{*}{ Tipo polínico } & \multicolumn{10}{|c|}{ Proporções } \\
\hline & $\mathbf{0 , 3}$ & $\mathbf{0 , 5}$ & 0,8 & 1,0 & 1,7 & 2,0 & 2,5 & 3,3 & 5,0 & 8,3 \\
\hline Baccharis & 0,0 & 0,0 & 0,0 & 0,9 & 0,0 & 0,8 & 0,0 & 0,0 & 0,0 & 0,0 \\
\hline Cecropia & 12,0 & 18,9 & 1,0 & 14,0 & 1,0 & 12,8 & 2,0 & 12,8 & 6,8 & 5,0 \\
\hline Gochnatia & 0,8 & 2,9 & 0,8 & 0,9 & 0,0 & 1,0 & 0,0 & 0,8 & 0,0 & 0,8 \\
\hline Myrcia & 0,8 & 5,5 & 2,8 & 8,0 & 1,0 & 5,0 & 2,8 & 2,8 & 2,8 & 6,8 \\
\hline Scrophulariaceae & 0,8 & 9,9 & 6,0 & 5,5 & 1,0 & 6,8 & 1,0 & 6,0 & 1,0 & 1,0 \\
\hline Vernonia & 0,8 & 5,9 & 1,0 & 2,0 & 1,0 & 0,8 & 0,8 & 0,8 & 0,0 & 0,0 \\
\hline
\end{tabular}

da UFV, Geraldo Neri Ferreira, pela competente contribuição no manejo das colméias. A CAPES e ao CNPq pela concessão de bolsas.

\section{Literatura citada}

Almeida-Muradian, L.B., Pamplona, L.C., Coimbra, S. \& Barth, O.M. 2005. Chemical composition and botanical evaluation of dried bee pollen pellets. Journal of food composition and analysis 18: 105-111.

Barth, O.M. 1989. O pólem no mel brasileiro. Gráfica Luxor, Rio de Janeiro.

Barth, O.M. 2004. Melissopalynology in Brazil: a review of pollen analysis of honeys, propolis and pollen loads of bees. Scientia Agricola 61: 342-350.

Biesmeijer, J.C., Marwijk, B. van, Deursen, K. van, Punt, W. \& Sommeijer, M.J. 1992. Pollen sources for
Apis mellifera L. (Hym, Apidae) in Surinam, based on pollen grain volume estimates. Apidologie 23: 245-256.

Castagnino, G.L.B., Message, D., Marco Júnior, P. \& Fernandes Filho, E.I. 2004. Avaliação da eficiência nutricional do substituto de pólen por meio de medidas de áreas de cria e pólen em Apis mellifera. Revista Ceres 41: 307-315.

Erdtman, G. 1960. The acetolysis method. A revised description. Svensk Botanisk Tidskrift, Stockolm 39: 561-564.

Luz, C.F.P. da, Barth, O.M., Cano, C.B.C., Felsner, M.L., Cruz-Barros, M.A.V., Guimarães, M.I.T.M. \& Correa, A.M.S. 2007a. Origem botânica do mel e derivados apícolas e o controle de qualidade. In: L.M. Barbosa \& N.A. Santos Junior (orgs.). A Botanica no Brasil: pesquisa, ensino e politicas públicas ambientais. Sociedade Botânica do Brasil, São Paulo, pp. 592-595. 
Luz, C.F.P., Thomé, M.L.\& Barth,O.M. 2007b. Recursos tróficos de Apis mellifera (Hymenoptera, Apidae) na região de Morro Azul do Tinguá, estado do Rio de Janeiro. Revista Brasileira de Botânica 30: 27-34.

Maurizio, A. \& Louveaux, J. 1965. Pollens de plantes mellifères dÉurope. U.G.A.F., Paris.

Melhem, T.S., Cruz-Barros, M.A.V., Corrêa, A.M.S., Makino-Watanabe, H., Silvestre-Capelato, M.S.F.\&

Esteves, V.L.G. 2003. Variabilidade polínica em plantas de Campos de Jordão (São Paulo, Brasil). Boletim do Instituto de Botânica 16: 1-104.

Modro, A.F.H., Message, D., Luz, C.F.P. \& Meira-Neto, J.A.A. 2007. Composição e qualidade de pólen apícola coletado em Minas Gerais. Pesquisa Agropecuária Brasileira 42: 1057-1065.
R Core Team. 2006. R development core team R: A language and environment for statistical computing. Foundation for Statistical Computing, Vienna.

Roubik, D.W. \& Moreno P., J.E. 1991. Pollen and spores of Barro Colorado island. Monograph in Systematic Botany 36: 1-268.

Salgado-Labouriau, M.L. 1973. Contribuição à palinologia dos cerrados. Academia Brasileira de Ciências, Rio de Janeiro.

Silveira, F.A. da. 1991. Influence of pollen grain volume on the estimation of the relative importance of its source to bees. Apidologie 22: 495-502.

Tasei, J.N. 1973. Comportement de nidification chez Osmia (Osmia) cornuta Latr et Osmia (Osmia) rufa L. (Hymenoptera, Megachilidae). Apidologie 4: 195-225. 